\title{
Static and Dynamic Forces on a Permanent Magnet Levitating Between Superconducting Surfaces
}

\author{
H. Barowski, K. M. Sattler, and W. Schoepe \\ Institut für Angewandte Physik, Universität Regensburg, D-8400 Regensburg, Germany
}

(Received March 15, 1993; revised June 2, 1993)

A charged magnetic microsphere (radius $100 \mu \mathrm{m}$ ) is levitating inside a superconducting niobium capacitor. Because of its charge of about $1 \mathrm{pC}$, oscillations about the equilibrium position can be excited and detected electrically. The properties of this oscillator are investigated in order to study the static and dynamic forces of magnetic levitation. We find resonance frequencies between $200 \mathrm{~Hz}$ and $600 \mathrm{~Hz}$. The resonance frequency and the damping are amplitude dependent due to nonlinear return forces and nonlinear friction, respectively. At small amplitudes the $Q$ value is about $10^{6}$ in vacuum. $A d c$ field can be applied to change the equilibrium position and consequently the resonance frequency. From the data the spatial dependence of the static force and of the dynamic stiffness can be determined and an empirical relation between both is established. Quite often, we find a hysteretic behavior of the static force whereas the stiffness is a reversible function of the position. Amplitude dependent damping is observed both in the decay of the free oscillations and in the dependence of the amplitude of the forced oscillations on the driving force.

\section{INTRODUCTION}

The physics of magnetic levitation is of considerable interest, both from a fundamental point of view and for technical applications. The force between a permanent magnet and a type II superconductor is a result of shielding currents of and the distribution and possible motion of flux lines. It therefore also depends on how the levitation status is prepared, i.e., whether the superconductor is cooled in zero field or in the presence of the magnet. Because of these complications a detailed quantitative understanding of the measured forces has not yet emerged. In several recent experiments on high- $\mathrm{T}_{c}$ superconductors ${ }^{1-4}$ the following general features have been observed and seem to be of fundamental significance. The static levitation force $F_{l}$ on the magnet as a function of the distance to the super- 
conducting surface is hysteretic, resulting in a "major hysteresis loop" both for the zero-field cooled and the field-cooled levitation. Small reversals of the motion of the magnet at various positions lead to "minor hysteresis loops" which are steeper than the major loop. From oscillations of the magnet about its equilibrium position the "dynamic stiffness" $m \omega^{2}$ ( $m$ is the mass of the magnet and $\omega / 2 \pi$ is the resonance frequency) can be determined which is different from the slopes of both major and minor loops. From the dynamic stiffness we define a "dynamic force" $F_{d}$ by $d F_{d} / d x=-m \omega \omega^{2}$. The relation between the static force and the dynamic force is not yet known.

In addition to these two forces the damping of the oscillations of the magnet is of interest. If normal conducting material, where eddy currents may cause dissipation, is far away from the magnet the damping in vacuum will only be due to losses inside the superconductor, i.e., flux motion and quasiparticles at finite $T / T_{c}$. So far, experimental investigation of the dissipative effects in levitation has been very scarce. ${ }^{5}$ From vibrating superconducting reeds, however, it is known that flux flow can lead to nonlinear and hysteretic frictional forces. ${ }^{6}$ Therefore, non-viscous damping can be expected and has been observed. ${ }^{5}$

In our present work we have made an attempt to study these problems experimentally in more detail. Our method consists of an investigation of the oscillations of a small permanent magnet levitating inside a superconducting capacitor. The method and its first results have been presented earlier. ${ }^{7,8}$ Here we wish to communicate a more complete study of the static and dynamic forces as well as of the frictional forces which all act on the levitating magnet and lead to interesting nonlinearities of the oscillator. From the data, we can determine both the dynamic force and the static force as a function of the distance between magnet and superconducting surface. We find an empirical relation between both forces. Furthermore, from the freely decaying oscillations and from the dependence of the amplitude of the forced oscillations on the drive we find particular power-laws of the velocity dependent frictional forces.

\section{EXPERIMENTAL DETAILS}

Our measuring cell consists of a parallel-plate capacitor made of niobium and having a spacing of $1 \mathrm{~mm}$. It contains a piece of $\mathrm{SmCo}_{5}$ which has been ground to spherical shape of $200 \mu \mathrm{m}$ diameter. From the density of the material we calculate that the particle has a mass $m=2.1 \cdot 10^{-8} \mathrm{~kg}$. From the specified remanence of the material ( 0.57 Tesla) we estimate that the magnetic dipole moment of the sphere is $2 \cdot 10^{-6} \mathrm{~A} \cdot \mathrm{m}^{2}$ (assuming a homogeneous magnetization). The magnetic field at the surface of the sphere therefore varies between 0.4 Tesla on the poles and 0.2 Tesla on the equator. 
During cool-down a large dc voltage $(\approx 700 \mathrm{~V})$ is applied to the bottom electrode. This causes a charge of about $1 \mathrm{pC}$ to flow onto the particle and it starts moving up and down between the electrodes until the temperature has dropped below $T_{c}=9.2 \mathrm{~K}$. Shielding currents on the superconductor repel the magnet and it comes to rest at an equilibrium position where gravitation and electrostatic forces are canceled by magnetic forces. This position is in general not in the middle of the capacitor, instead the data indicate that the particle rests closer to one or the other of the two electrode surfaces. By applying a dc voltage to the capacitor the equilibrium position of the magnet can be varied over a limited range $(\approx 40 \mu \mathrm{m})$. Although the equilibrium position cannot be measured directly its changes due to the dc voltage can be implied from the variation of the frequency with which the sphere oscillates, see below.

The oscillations of the particle are excited and detected by means of the electronics shown schematically in Fig. 1. When the particle moves at a velocity $v$ normal to the capacitor plates the preamplifier detects a current $I$ which is given by

$$
I=q v / d
$$

where $q$ is the charge of the particle and $d=1 \mathrm{~mm}$ is the spacing. Assuming harmonical vibration the velocity of the oscillating particle is determined by the amplitude $A$ and the resonance frequency $f=\omega / 2 \pi$, i.e., we have

$$
I=q \omega A / d
$$

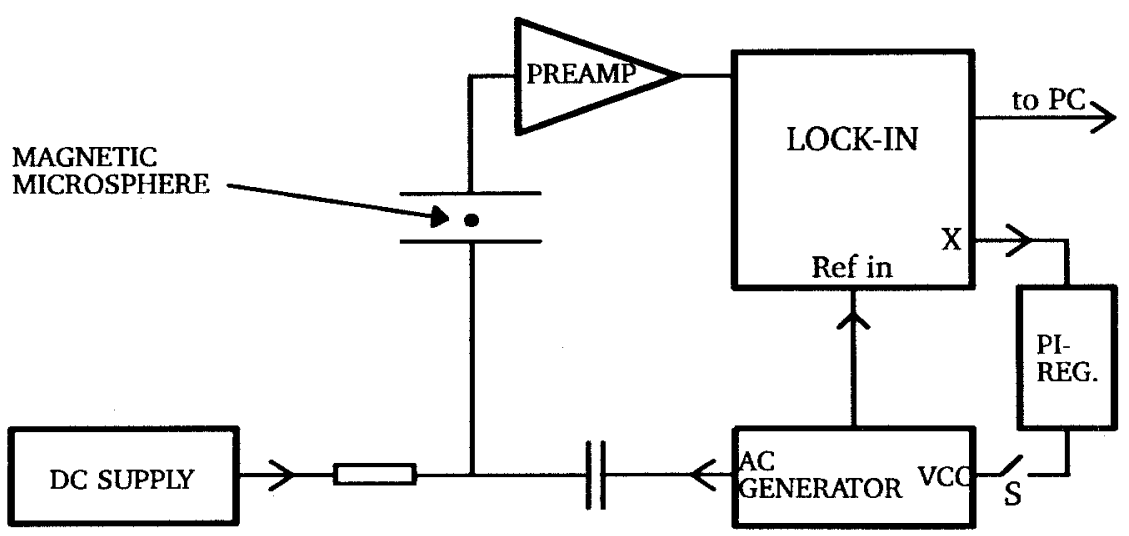

Fig. 1. Experimental set-up. The charged magnetic sphere (diameter $0.2 \mathrm{~mm}$ ) is floating inside a superconducting niobium capacitor (diameter $4 \mathrm{~mm}$, spacing $1 \mathrm{~mm}$ ) at $4.2 \mathrm{~K}$. The switch $\mathrm{S}$ can be closed for a fixed phase relation between ac drive and oscillation. 
We usually have $q \approx 1 \mathrm{pC}$ and $f \approx 300 \mathrm{~Hz}$, hence a current amplitude of $1 \mathrm{pA}$ corresponds to $v \approx 1 \mathrm{~mm} / \mathrm{s}$ and $A \approx 0.5 \mu \mathrm{m}$. The oscillations are excited by an ac voltage in the millivolt range. A regulator can be used to fix the phase between the oscillations and the generator by nulling one output channel of the two-phase lock-in amplifier. For a quantitative analysis of the forced oscillations the capacitively coupled pick-up which is superimposed upon the signal of the sphere has to be subtracted numerically by standard complex analysis. All data to be presented in this work were obtained with an evacuated capacitor at a temperature of $4.2 \mathrm{~K}$.

\section{RESULTS AND DISCUSSION}

\subsection{Static and Dynamic Forces}

Oscillations of the levitating particle can be excited by applying an ac voltage to the capacitor. The resonance frequency, which typically lies in the range from $200 \mathrm{~Hz}$ to $600 \mathrm{~Hz}$, depends on the equilibrium position which can be varied by a dc voltage $U_{d c}$ between the capacitor plates, see Fig. 2. With no exception, we always obtain a straight line when plotting the square of the frequency versus voltage. ${ }^{9}$ This empirical result will be of significance in our analysis, see below. The sign of the slope depends on the sign of the charge and on whether the particle is levitating just above the bottom plate or suspending just below the upper plate. In any case, we

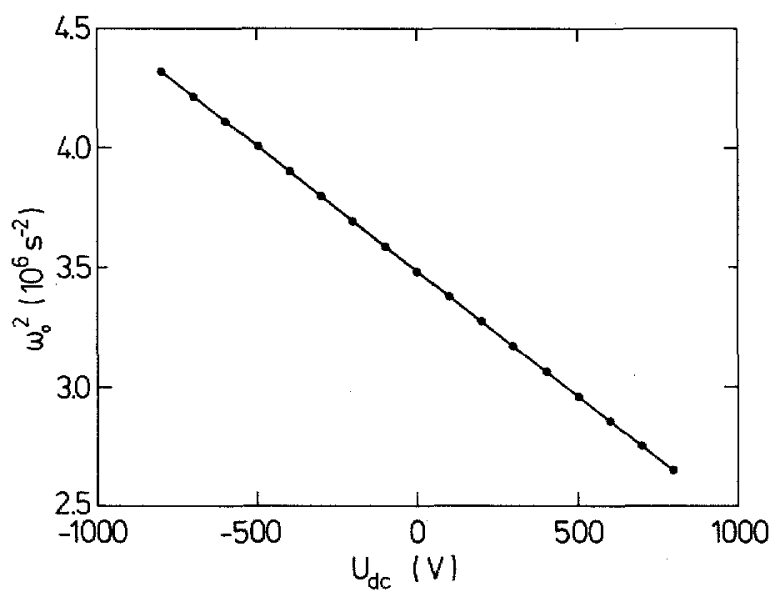

Fig. 2. Resonance frequency of the magnet as a function of the dc voltage between the capacitor plates. We always observe a linear dependence of the square of the frequency. The charge of the oscillating sphere is $q=-1.1 \mathrm{pC}$. 
interpret an increase of the resonance frequency as a decrease of the distance to the adjacent superconducting surface.

At each equilibrium position we measure the following typical nonlinear properties of the oscillations. Firstly, we determine the shift of the resonance frequency $\Delta f=f-f_{0}$ with increasing amplitude, i.e.,

$$
\Delta f / f_{0}=-a I^{2}
$$

where $f_{0}$ is the resonance frequency for $I \rightarrow 0$, and $a$ is positive, i.e., we always find a negative shift. Details of the resonance curves have been presented earlier, ${ }^{8}$ here we focus on the dependence of the coefficient $a$ on the equilibrium position (i.e., on the voltage or the corresponding frequency), see Fig. 3. Simultaneously, we measure the amplitude of the second harmonic $I_{2 \omega}$ which varies in proportion to $I^{2}$ :

$$
I_{2 \omega}=\varepsilon I^{2}
$$

In Fig. 4 the frequency dependence of $\varepsilon$ is depicted.

From the coefficients $a$ and $\varepsilon$ we can determine the nonlinear coefficients $\alpha$ and $\beta$ of the dynamical return force $F$ at each equilibrium position $x_{0}$ :

$$
F / m=-\omega_{0}^{2}\left(x_{0}\right)\left(x-x_{0}\right)-\alpha\left(x_{0}\right)\left(x-x_{0}\right)^{2}-\beta\left(x_{0}\right)\left(x-x_{0}\right)^{3}
$$

(Note that this return force is the third order Taylor expansion of the dynamic force $F_{d}$ at the equilibrium position $x_{0}$.) It is known, ${ }^{10}$ that (5) leads to an amplitude dependent frequency shift

$$
\Delta f / f_{0}=\left(3 \beta / 8 \omega_{0}^{2}-5 \alpha^{2} / 12 \omega_{0}^{4}\right) A^{2}
$$

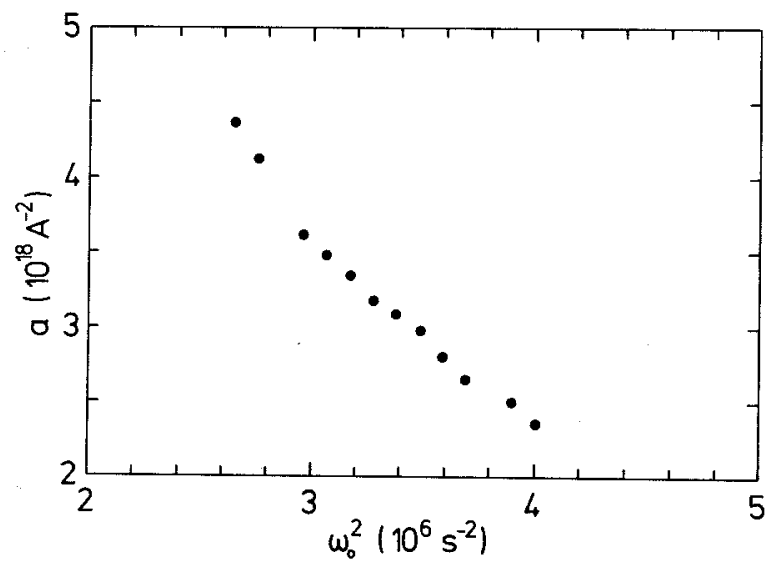

Fig. 3. The coefficient $a$ of the amplitude dependent frequency shift, see Eq. (3), at various resonance frequencies depending on the applied dc voltage. 


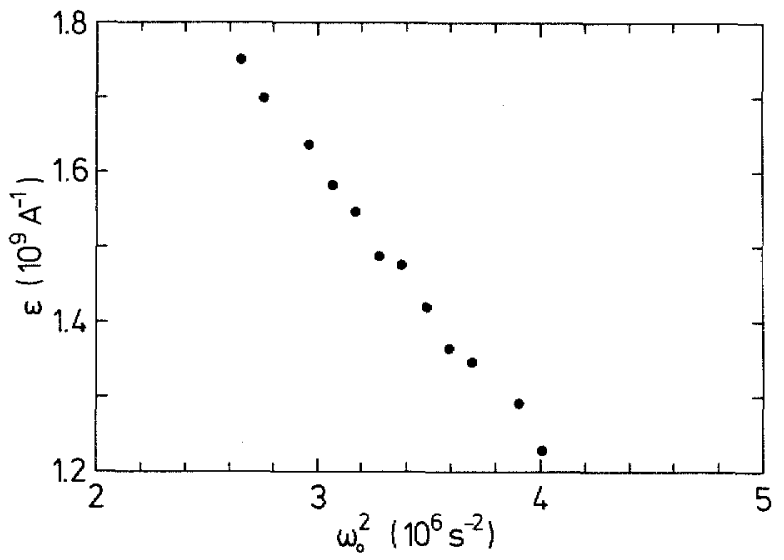

Fig. 4. The coefficient $\varepsilon$ of the amplitude dependent secondharmonic, see Eq. (4), at various resonance frequencies.

and to a second-harmonic amplitude $A_{2 \omega}$ being given by

$$
A_{2 \omega}=\left(\alpha / 6 \omega_{0}^{2}\right) A^{2}
$$

Hence, from $\varepsilon\left(\omega_{0}^{2}\right)$ and $a\left(\omega_{0}^{2}\right)$ we can determine $\alpha\left(\omega_{0}^{2}\right)$ and $\beta\left(\omega_{0}^{2}\right)$, if the charge $q$ of the sphere is known. The determination of $q$ will be discussed in the Appendix. In the following we use the measured value $q=-1.1 \mathrm{pC}$. Because $\omega_{0}^{2}, \alpha$, and $\beta$ are given by the first, second and third derivative of $F_{d}$ at every equilibrium position $x=x_{0}$, the following relations must hold:

$$
\begin{aligned}
d \omega_{0}^{2} / d x & =2 \alpha \\
d \alpha / d x & =3 \beta
\end{aligned}
$$

and therefore

$$
\frac{d \alpha^{2}}{d \omega_{0}^{2}}=\frac{d \alpha}{d \omega_{0}^{2}} \cdot 2 \alpha=\frac{d \alpha}{d \omega_{0}^{2}} \cdot \frac{d \omega_{0}^{2}}{d x}=\frac{d \alpha}{d x}=3 \beta
$$

We find a linear dependence of $\alpha^{2}$ on $\omega_{0}^{2}$ and a constant $\beta$, in agreement with (10). From (8) we can calculate the spatial dependence $\omega_{0}^{2}(x)$ by solving for $\Delta x$ between two $\omega_{0}^{2}$ values and the corresponding $\alpha$ values:

$$
\omega_{02}^{2}-\omega_{01}^{2}=\left(2 \alpha_{2}+2 \alpha_{1}\right) \cdot \frac{\Delta x}{2}+\cdots
$$

neglecting higher order terms because $\beta=$ const. The result is shown in Fig. 5. The total variation of the equilibrium position due to the dc voltage 


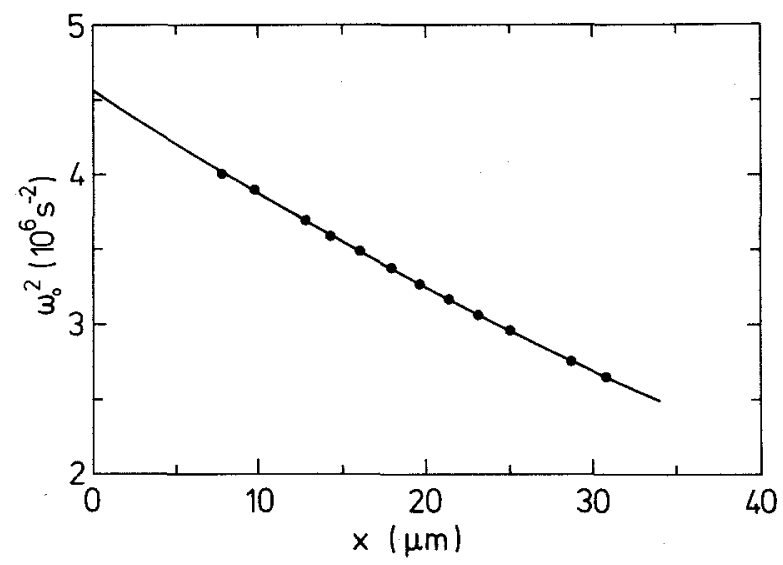

Fig. 5. Spatial variation of the resonance frequency calculated from Eq. (11). (The position $x=0$ is arbitrary.)

is only about $25 \mu \mathrm{m}$. Larger variations require larger voltages than the $\pm 800 \mathrm{~V}$ in this experiment. The spatial resolution, however, is very high: a frequency resolution of $10 \mathrm{mHz}$ corresponds to a spatial resolution of $3 \mathrm{~nm}$. From $\omega_{0}^{2}(x)$ we can convert the measured frequency dependences of the Taylor coefficients $\alpha$ and $\beta$ into spatial dependences. Furthermore, we can compute the dynamic force to third order accuracy by a numerical integration $^{11}$ :

$$
\Delta F_{d} / m=\left(\omega_{01}^{2}+\omega_{02}^{2}\right) \cdot \frac{\Delta x}{2}+\left(2 \alpha_{1}-2 \alpha_{2}\right) \frac{\Delta x^{2}}{10}+\left(6 \beta_{1}+6 \beta_{2}\right) \frac{\Delta x^{3}}{120}
$$

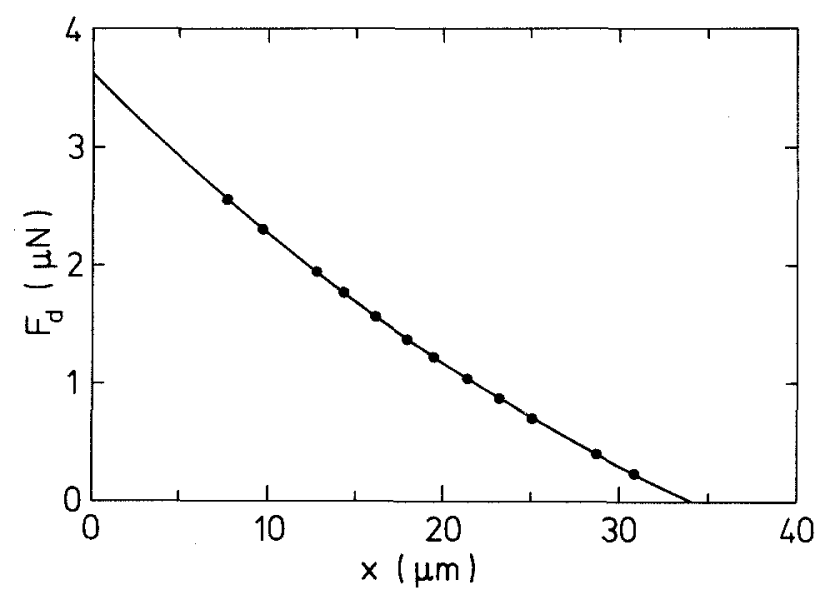

Fig. 6. Variation of the dynamic force on the levitating magnet calculated from Eq. (12). (The zero-points are arbitrary.) 
see Fig. 6, where we choose $F_{d}=0$ at an arbitrary value of $x$. (Note that $F_{d}$ is defined only within an arbitrary additive constant.) Because of the constant values of $\beta$ the dynamic force has terms up to third power in $x$ over our accessible spatial interval.

Next, we analyze the static levitation force $F_{l}$ which together with the external forces $m g$ (gravitation) and $q \mathscr{E}\left(\mathscr{E}=U_{d c} / d\right.$ is the external electrostatic field) determines the equilibrium position:

$$
\vec{F}_{l}(x)+m \vec{g}+q \overrightarrow{\mathscr{E}}=0
$$

From the field dependent frequency (Fig. 2) and the spatial dependence of the frequency (Fig. 5) we obtain $F_{l}(x)$ from (13), see Fig. 7. It is obvious that the static force has less spatial variation than the dynamic force, i.e., the dynamic stiffness is larger than the slope of the static force. From Fig. 2 we empirically find $\omega_{0}^{2}$ to vary linearly with field. Therefore, we make the following Ansatz:

$$
\omega_{0}^{2}(\mathscr{E})=\omega_{00}^{2}-c(m g+q \mathscr{E})
$$

where $\omega_{00}^{2}$ and $c$ are constants. Inserting (13) we obtain

$$
\omega_{0}^{2}(\mathscr{E})=\omega_{00}^{2}+c F_{l}(x(\mathscr{E}))
$$

or

$$
-\frac{1}{m} \frac{d F_{d}(x)}{d x}=\omega_{00}^{2}+c F_{l}(x)
$$

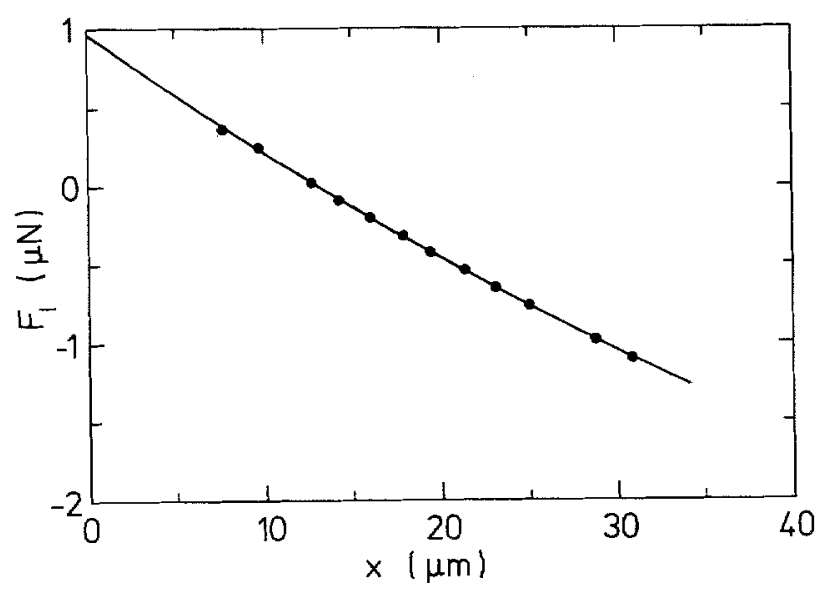

Fig. 7. Variation of the static levitation force calculated from Eq. (13). 
Equations (15) or (16) establish an empirical relation between both forces. At present, we have no physical model from which (16) could be deduced rigorously. It is interesting to note, however, that Eq. (15) is reminiscent of a vibrating string whose frequency squared is proportional to the tension.

\subsection{Hysteresis of the Static Levitation Force}

So far, we have investigated about 50 oscillators carrying different charges and having different resonance frequencies. All of them have qualitatively the same dynamic behavior as described above. The static properties, however, can differ in a very remarkable way: about $60 \%$ of our oscillators exhibit hysteretic behavior in the $\omega_{0}^{2}\left(U_{d c}\right)$ diagram, as shown in Fig. 8. After increasing the voltage up to $+1000 \mathrm{~V}$ (point B) we observe that a reduction of the voltage causes $\omega_{0}^{2}$ to drop along a different path to point $C$, where the slope changes back to the initial one. At point $D$ $(-1000 \mathrm{~V})$ the voltage is reversed again and $\omega_{0}^{2}$ increases slowly until point $A$ is reached. Further increase of the voltage changes $\omega_{0}^{2}$ back to point B. This loop ABCDA is observed for counter-clockwise circulation. If the voltage is reversed earlier (i.e., before $+1000 \mathrm{~V}$ or $-1000 \mathrm{~V}$ are reached) new paths which are parallel to $\mathrm{BC}$ or DA are observed between the limiting lines $\mathrm{AB}$ or $\mathrm{CD}$. All these shallower paths are perfectly reversible whereas the steep limiting lines $\mathrm{AB}$ and $\mathrm{CD}$ can be observed only for

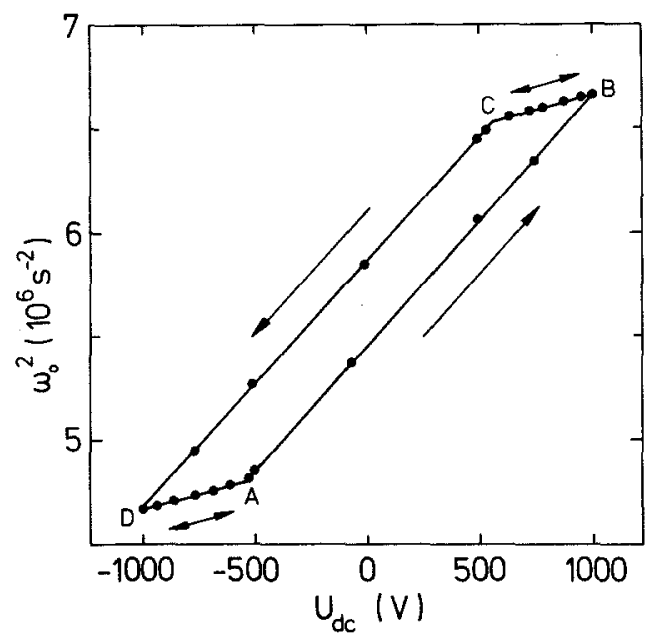

Fig. 8. Hysteretic behavior of the resonance frequency (i.e., of the equilibrium position). Branches $\mathrm{AB}$ and $\mathrm{CD}$ are irreversible whereas $\mathrm{BC}$ and $\mathrm{DA}$ are reversible. The charge of this oscillator is $q=+1.1 \mathrm{pC}$. 
increasing or decreasing frequency, respectively. Stability checks over many hours reveal that the frequency of the irreversible lines is slowly drifting into the original loop. Although the drift $(\sim 0.1 \mathrm{~Hz} /$ hour $)$ is slowing down in time it never really stops. (This behavior is probably related to the known logarithmic relaxation of the magnetization.) Thus the hysteresis loop appears to shrink gradually, probably into a single stable and reversible line.

Another most remarkable property of the hysteresis loop is that the nonlinear dynamical properties (amplitude dependent frequency shift $a$ in (3) and second-harmonic coefficient $\varepsilon$ in (4)) are found to depend only on $\omega_{0}^{2}$ and not on the particular branch of the loop. This implies that the dynamical force is a continuous single-valued function of the position without any hysteretic behavior. Going through the same analysis as described in the previous chapter we obtain from the data of Fig. 8 the hysteresis loop of the static force $F_{l}$, see Fig. 9. Note that the reversible paths are now the steeper ones because

$$
\frac{d \omega_{0}^{2}}{d \mathscr{E}}=\frac{d \omega_{0}^{2}}{d x} \cdot \frac{d x}{d \mathscr{E}}=2 \alpha(x) \cdot \frac{d x}{d \mathscr{E}}
$$

which means that a small slope in Fig. 8 is equivalent to a small change of position, i.e., to a stiff static force. Finally, it should be mentioned that the

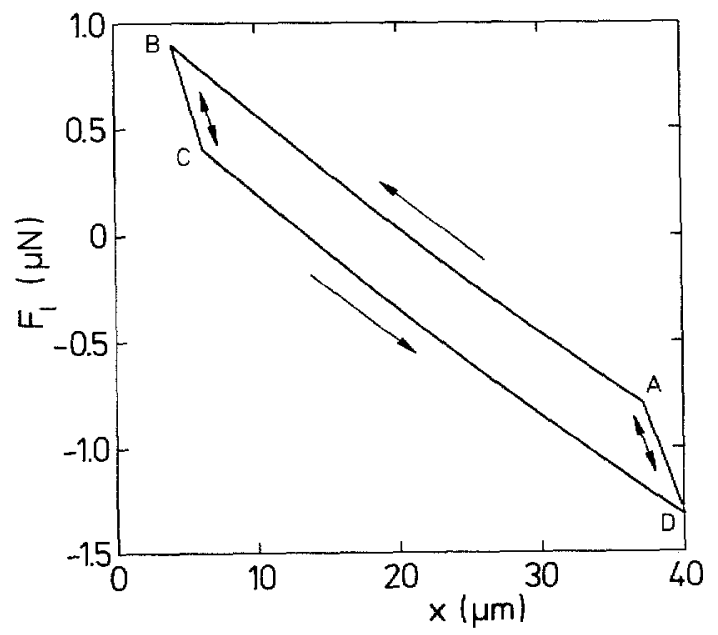

Fig. 9. Hysteretic static force resulting from the data of Fig. 8. Note that $F_{l}$ is much stiffer on the reversible paths than on the irreversible ones. The dynamic stiffness, which is not hysteretic, has intermediate values, see text. (The position $x=0$ is arbitrary.) 
dynamical stiffness $-d F_{d} / d x$ is larger than the slope of $F_{l}$ along the irreversible branches $A B$ and $C D$, but smaller than the slopes of the reversible sections $\mathrm{BC}$ and $\mathrm{AD}$. This behavior agrees with earlier experiments on bigger magnets above high- $\mathrm{T}_{c}$ superconductors ${ }^{1-4}$ as discussed in the Introduction.

\subsection{Frictional Forces}

The damping mechanisms can be studied either by recording the time dependence of the amplitude of the freely decaying oscillations or by analyzing the amplitude of the forced oscillations as a function of the drive. The usual case of a linear frictional force $F_{f} \propto v$ leads to an exponential decay of the free oscillations and to a linear increase of the amplitude of the forced oscillations with the amplitude of the external driving force $F_{\text {ext }}=q \mathscr{E}_{a c} \sin (\omega t)$, where $\mathscr{E}_{a c}=U_{a c} / d$. This behavior was observed only at small amplitudes $A \approx 1 \mu \mathrm{m}$ [8]. For larger amplitudes nonlinear friction $F_{f} \propto|v|^{r}$ with $1<r \leqslant 2$ is observed. It is easy to show (see Appendix) that this nonlinear friction leads to an amplitude which increases as $U_{a c}^{1 / r}$ and to a decay of the free oscillations with time $t$ being given by $A^{-(r-1)} \propto t$.

In Fig. 10 a free decay is depicted which can be described by nonlinear friction with $r=1.5$ at large amplitudes as can be seen from the linear

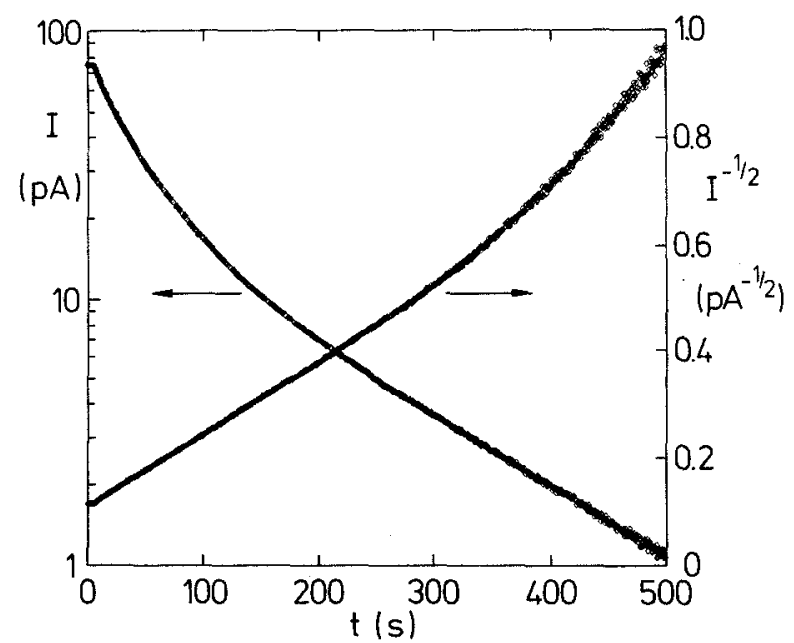

Fig. 10. Free decay of the oscillation. Left scale is the usual semi-logarithmic plot; small amplitudes decay exponentially. Right scale: same data plotted on a $I^{-0.5}$ reciprocal scale; note the straight-line behavior at large amplitudes. The charge of this oscillator is $q=-1.3 \mathrm{pC}$ and the resonance frequency is $f_{0}=340 \mathrm{~Hz}$. 


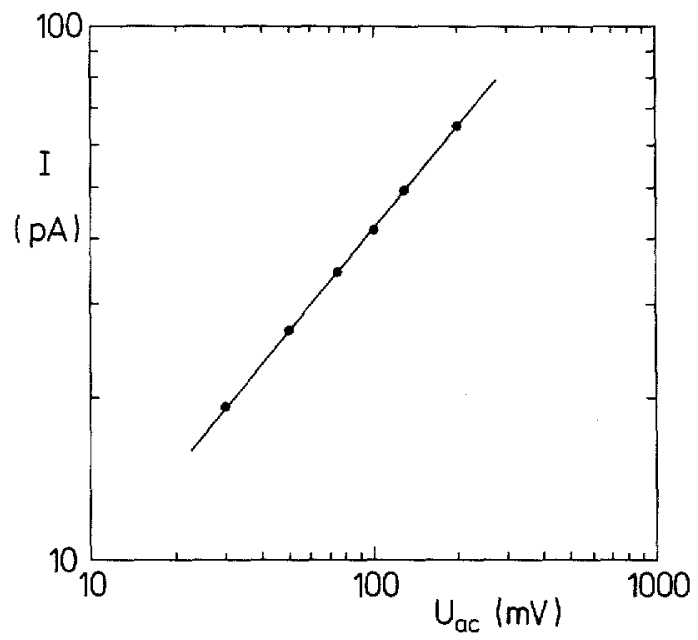

Fig. 11. Forced oscillation amplitude versus drive; same oscillator as in Fig. 10. The slope of the straight line indicates a $U_{a c}^{0.66}$ law, which is in agreement with the nonlinear friction deduced from Fig. 10.

increase of $I^{-0.5}$ and by linear friction at low amplitudes where $\ln (I)$ has a straight-line behavior. The corresponding response of the forced oscillator at large drive is shown in Fig. 11. The amplitude is found to increase as $U_{a c}^{0.66}$ in agreement with the free decay in Fig. 10. In the regime of linear friction we obtain the Q-value from the decay time $\tau$ in Fig. 10:

$$
Q=\frac{\omega \tau}{2}=1.8 \cdot 10^{5}
$$

This yields the coefficient $\hat{\lambda}$ of the linear frictional force $F_{f}=-m \lambda v$ from $\lambda=\omega / Q=2 / \tau=1.8 \cdot 10^{-2} s^{-1}$. In the Appendix it is shown how the friction coefficient can be determined from the data in case of a nonlinear frictional force.

In general, each oscillator had its own nonlinear friction $1<r \leqslant 2$. Rather often a quadratic dependence $r=2$ could be observed, as reported earlier. ${ }^{7,8}$ The origin of this variation is probably due to the particular distribution of shielding currents and flux lines in the superconductor close to the magnet. Eddy currents in the quasiparticle system (note that $T / T_{c}=$ $4.2 / 9.2=0.46$ ) are generated by the time-dependent magnetic field of the oscillating magnet. They give rise to linear damping. In addition, motion of flux lines will cause dissipation, which is known to be viscous (i.e., linear) for small displacements and hysteretic (i.e., nonlinear) for large-amplitude 
oscillations because of irreversible jumps of the flux lines, ${ }^{6,12}$ leading to an amplitude dependent damping with $1.1 \leqslant r \leqslant 2$.

The damping of the oscillating magnet can be influenced by changing the equilibrium position with a static electric field. The following general features were observed. Pushing the magnet closer to the superconducting surface (as indicated by an increase of the resonance frequency) leads to more linear friction: at large amplitudes the values of $r$ tend to decrease towards $r=1$, and the small-signal Q-values drop, e.g., from $10^{6}$ to $10^{5}$ when the frequency is changed from $170 \mathrm{~Hz}$ to $300 \mathrm{~Hz}^{13}$ It is plausible that this behavior is caused by the increase of the time-dependent magnetic field at the niobium surface. A quantitative description of the measured dissipation in terms of the above mechanisms, however, appears to be rather complicated and is presently not available. Further experiments, including a variation of $T / T_{c}$, are needed to shed more light on the damping effects.

\section{CONCLUSION}

Our experimental results demonstrate the profound differences between static and dynamic forces on the levitating magnet. An empirical relation between these forces is established: the dynamic stiffness is a linear function of the static force. In addition, while the static force may be hysteretic the dynamic force is always found to be reversible within the investigated interval of equilibrium positions.

The attenuation of the oscillations of the magnet about its equilibrium position gives evidence of nonlinear friction at large amplitudes which may be attributed to irreversible flux motion. In agreement with vibrating reed experiments ${ }^{6}$ we find nonlinear frictional forces which can be described by power laws of the velocity (or amplitude) $v^{r}$ up to $r=2$. At low amplitudes we find linear friction which may be caused by viscous effects in the flux lattice and by normal eddy currents. A quantitative description of the dissipation is not yet available.

Further experiments are planned to investigate the levitational forces at different temperatures and in external magnetic fields. Of course, various substrates, including high $-\mathrm{T}_{c}$ superconductors, will also be used. In addition to studying the physics of magnetic levitation these oscillators can be applied for low temperature viscometry in helium because of their low residual damping. ${ }^{15}$ Furthermore, they can be used for investigating critical velocities and vorticity in superfluid helium. ${ }^{16}$ 


\section{APPENDIX 1}

We calculate the time dependent amplitude of the freely decaying oscillations due to a nonlinear drag force $F_{f}=-m \gamma \dot{x}|\dot{x}|^{r-1}$ by standard techniques. The equation of motion is

$$
\ddot{x}+\omega_{0}^{2} x=f(x, \dot{x})
$$

where

$$
f(x, \dot{x})=-\alpha x^{2}-\beta x^{3}-\gamma \dot{x}|\dot{x}|^{r-1}
$$

Using the Ansatz $x=A \cos \psi$ the time dependence of the amplitude $A$ can be found from ${ }^{14}$

$$
\dot{A}=-\frac{1}{2 \pi \omega_{0}} \int_{0}^{2 \pi} f\left(A \cos \psi,-A \omega_{0} \sin \psi\right) \sin \psi d \psi
$$

The only contribution comes from the drag force and gives

$$
\dot{A}=\frac{-\gamma \omega_{0}^{r-1} A^{r}}{2 \pi} \int_{0}^{2 \pi}|\sin \psi|^{r-1} \sin ^{2} \psi d \psi
$$

which can be written as

$$
\dot{A}=\frac{-\gamma \omega_{0}^{r-1}}{\pi} A^{r} \int_{0}^{\pi} \sin ^{r+1} \psi d \psi=-C_{r} A^{r}
$$

where

$$
C_{r}=\gamma \omega_{0}^{r-1} G_{r}
$$

and

$$
G_{r}=\frac{1}{\pi} \int_{0}^{\pi} \sin ^{r+1} \psi d \psi
$$

The solution of the differential equation is (for $r>1$ )

$$
A^{-(r-1)}(t)=(r-1) C_{r} t+\text { const. }
$$

In case of a quadratic frictional force $r=2$ this gives

$$
A^{-1}(t)=A^{-1}(0)+\left(4 \gamma \omega_{0} / 3 \pi\right) t
$$

The stationary amplitude of the forced oscillations is most easily calculated from the energy balance. At resonance the external driving force $F_{\text {ext }}$ and the velocity $\dot{x}=-A \omega_{0} \sin \omega_{0} t$ are in phase, i.e., (for $q>0$ )

$$
F_{\text {ext }}=-q \frac{U_{a c}}{d} \sin \omega_{0} t
$$


Therefore, the energy balance can be expressed simply by

$$
\frac{1}{T} \int_{0}^{T} F_{\mathrm{ext}} \cdot \dot{x} \cdot d t=\frac{1}{T} \int_{0}^{T} F_{f} \cdot \dot{x} \cdot d t
$$

from which we find

$$
A^{r}=\left(q / 2 d \gamma m \omega_{0}^{r} G_{r}\right) \cdot U_{a c}
$$

If we express the amplitude $A$ in (A1) and (A2) by the measured current $I$ (see Eq. (2)) we finally obtain for the free oscillations

$$
I^{-(r-1)}(t)=I^{-(r-1)}(0)+D t
$$

where

$$
D=(r-1) \gamma G_{r}(q / d)^{-(r-1)}
$$

and for the forced oscillations we get

$$
I=b \cdot U_{a c}^{1 / r}
$$

where

$$
b=\left[(q / d)^{r+1} / 2 \gamma m G_{r}\right]^{1 / r}
$$

From measurements of the coefficients $b$ and $D$ we can determine the friction coefficient $\gamma$ and the charge $q$ of the magnetic sphere. For the case of a quadratic friction, e.g., a typical value is $\gamma \approx 1 \mathrm{~m}^{-1}$.

A similar analysis can be performed to calculate the frequency shift, see Ref. 14. The second-order result is given by Eq. (6). Effects of the drag force on the frequency shift are completely negligible in all of our cases.

\section{APPENDIX 2}

At the end of the experiment we measure the charge of the magnet by heating the capacitor above $\mathrm{T}_{c}$ and integrating the current pulse when the magnet falls down to the lower electrode. This charge is actually only the fraction $x_{0} / d$ of the real charge, where $x_{0}$ is the equilibrium position measured from the bottom electrode. Because we can determine the charge also from (A3) and (A4), the equilibrium position $x_{0}$ can be implied. The result, however, is not very accurate because the spacing $d$ of the capacitor is only a factor of 5 larger than the diameter of the sphere and the charge probably is correct only within $10 \%$. Nevertheless, we get reasonable 
values $0<x_{0} / d<1$ which tell us whether the magnet is levitating above the lower electrode or suspending below the upper one. The same conclusion can be reached from the sign of the measured charge and the slope of the $\omega_{0}^{2}\left(U_{d c}\right)$ lines (Fig. 2), because an increase of the frequency is understood as an equilibrium position closer to the adjacent surface.

\section{ACKNOWLEDGMENTS}

We are grateful to our colleagues P. Eizinger, K. Gloos, J. T. Simola, and J.T. Tuoriniemi for their co-operation in earlier stages of these experiments.

\section{REFERENCES}

1. F. C. Moon, K.-C. Weng, and P.-Z. Chang, J. Appl. Phys. 66, 5643 (1989).

2. P.-Z. Chang, F. C. Moon, J. R. Hull, and T. M. Mulcahy, J. Appl. Phys. 67, 4358 (1990).

3. B. R. Weinberger, L. Lynds, and J. R. Hull, Supercond. Sci. Technol. 3, 381 (1990).

4. S. A. Basinger, J. R. Hull, and T. M. Mulcahy, Appl. Phys. Lett. 57, 2942 (1990).

5. V. V. Nemoshkalenko, E. H. Brandt, A. A. Kordyuk, and B. G. Nikitin, Physica C 170, 481 (1990).

6. P. Esquinazi, J. Low Temp. Phys. 85, 139 (1991).

7. K. Gloos, J. H. Koivuniemi, W. Schoepe, J. T. Simola, and J. T. Tuoriniemi, Physica B, $165 \& 166,119(1990)$.

8. P. Eizinger, W. Schoepe, K. Gloos, J. T. Simola, and J. T. Tuoriniemi, Physica B 178, 340 (1992).

9. In Fig. 9 of Ref. 8 the voltage interval was too limited to observe a deviation from an apparently linear voltage dependence of the frequency.

10. L. D. Landau and E. M. Lifshitz, Lehrbuch der Theoretischen Physik, Vol. 1 (AkademieVerlag, Berlin, 1979).

11. R. Zurmühl, Praktische Mathematik für Ingenieure und Physiker (Springer-Verlag, Berlin, 1962).

12. E. H. Brandt, P. Esquinazi, and H. Neckel, J. Low Temp. Phys. 63, 187 (1986).

13. K. M. Sattler, Diploma thesis (Universität Regensburg, unpublished, 1992).

14. N. N. Bogoljubow and J. A. Mitropolski, Asymptotische Methoden in der Theorie der nichtlinearen Schwingungen (Akademie-Verlag, Berlin, 1965).

15. K. Gloos, W. Schoepe, J. T. Simola, and J. T. Tuoriniemi, Cryogenics 32, 791 (1992).

16. H. Barowski and W. Schoepe, to be published. 